\section{A implementação de Programas de Residência Médica em Medicina de Família e Comunidade em uma capital da Região Sudeste, Brasil: relato de experiência}

\author{
The implementation of Medical Residency Programs in Family \\ and Community Medicine in a capital of the Southeast Region, \\ Brazil: experience report
}

\author{
La implementación de Programas de Residencia Médica en \\ Medicina de Familia y Comunidad en una capital de la Región \\ Sudeste, Brasil: relato de experiencia
}

\section{Resumo}

Objetivo: Discutir o processo de implantação de três Programas de Residência Médica em Medicina de Família e Comunidade (PRM-MFC) em uma capital da Região Sudeste do Brasil na perspectiva da interface dos programas com a organização do sistema de saúde local. Método: Trata-se de um relato de experiência oriundo das vivências dos supervisores dos programas e da equipe técnica do município responsável pela gestão da integração ensino-serviço. Foi produzida uma narrativa significativa aos atores do processo em momento presencial coletivo. Uma matriz SWOT foi elaborada para elencar os fatores que influenciam a implementação dos PRM-MFC na rede de serviços. Resultados: Há três PRM-MFC em atividade no município, todos em estágio inicial de implementação, totalizando nove vagas de R1. Os residentes são beneficiados por uma rede de serviços bem estruturada, diversificada e informatizada, incluindo preceptores sensibilizados e um forte apoio multiprofissional. Contudo, há questões que dificultam esse processo, como a incipiente política de valorização da preceptoria, as insuficiências do modelo de atenção primária existente para a formação médica, as dificuldades na gestão compartilhada do processo e a pouca legitimidade da RM perante a comunidade e os trabalhadores do serviço. Conclusão: Há íntima relação entre o desenvolvimento da rede local de saúde, em particular da APS, com a qualidade do PRM. Assim, é fundamental ter em vista não apenas as questões pedagógicas, mas também todo um conjunto de dispositivos de gestão compartilhada e qualificação da rede de serviços.

Palavras-chave: Internato e Residência; Medicina de Família e Comunidade; Política de Saúde
Thiago Dias Sartia

Marcello Dala Bernardina Dalla ${ }^{b}$

Tânia Mara Machadoc

Mary Cristina França de Oliveira Fonsecac

Sandra Mara Soeiro Bofc

José de Almeida Castro Filhod

Mônica Cola Carriello Correa ${ }^{c}$

Sérgio Renato Miranda Torres ${ }^{c}$

Sheila Cristina de Souza Cruz ${ }^{\circ}$

a Universidade Federal do Espírito Santo
(UFES). Vitória, ES, Brasil.
thiagosarti@yahoo.com.br
(Autor correspondente)

b Secretaria de Estado da Saúde do Espírito Santo. Vitória, ES, Brasil. marcellodbdalla@gmail.com

c Escola Técnica do SUS, Secretaria Municipal de Saúde de Vitória. Vitória, ES, Brasil. gtestagiopesquisa@gmail.com

${ }^{\mathrm{a}}$ Médico de Família e Comunidade, Unimed Vitória. Vitória, ES, Brasil.

jcfnit@hotmail.com
Como citar: Sarti TD, Dalla MDB, Machado TM, Fonseca MCFO, Bof SMS, Castro Filho JA, et al. A implementação de Programas de Residência Médica em Medicina de Família e Comunidade em uma capital da Região Sudeste, Brasil: relato de experiência. Rev Bras Med Fam Comunidade. 2018;13(40):1-12. http://dx.doi.org/10.5712/rbmfc13(40)1684
Fonte de financiamento: declaram não haver.

Parecer CEP: não se aplica. Conflito de interesses: declaram não haver. Procedência e revisão por pares: revisado por pares. Recebido em: 30/01/2018. Aprovado em: 07/03/2018. 


\begin{abstract}
Objective: Discuss the implementation process of three Medical Residency Programs in Family and Community Medicine (PRM-MFC) in a capital city of the Southeast Region of Brazil, in the perspective of the interface of the programs with the organization of the local health system. Method: This is an experience report from the experiences of the program supervisors and the technical team of the municipality responsible for integration of teaching-service. A significant narrative to the actors of the process was produced in a collective presencial moment. A SWOT matrix was developed to list the factors that influence the implementation of PRM-MFC in the health service network. Results: There are three PRM-MFC in activity in the municipality, all in the initial stage of implementation, totaling nine vacancies of R1. Residents are benefited by a well-structured, diversified and computerized service network, including sensitized preceptors and strong multi-professional support. However, there are issues that complicate this process, such as the incipient preceptory valorization policy, the insufficiencies of the existing primary care model for medical training, the difficulties in the shared management of the process, and the lack of legitimacy of RM before the community and service workers. Conclusion: There is an intimate relationship between the development of the local health care network, in particular APS, with the quality of the PRM. Thus, it is essential to look not only at pedagogical issues, but also at the whole set of shared management devices and qualification of the service network.
\end{abstract}

Keywords: Internship and Residency; Family Practice; Health Policy

\title{
Resumen
}

Objetivo: Discutir el proceso de implantación de tres Programas de Residencia Médica en Medicina de Familia y Comunidad (PRM-MFC) en una capital de la Región Sudeste de Brasil en la perspectiva de la interfaz de los programas con la organización del sistema de salud local. Método: Se trata de un relato de experiencia oriundo de las vivencias de los supervisores de los programas y del equipo técnico del municipio responsable por la gestión de la integración enseñanza-servicio. Se produjo una narrativa significativa a los actores del proceso en un momento presencial colectivo. Una matriz SWOT fue diseñada para definir los factores que influencian la implementación de los PRM-MFC en la red de servicios. Resultados: Hay tres PRM-MFC en actividad en el municipio, todos en etapa inicial de implementación, totalizando nueve vacantes de R1. Los residentes se benefician de una red de servicios bien estructurada, diversificada e informatizada, incluidos los preceptores sensibilizados y un fuerte apoyo multiprofesional. Sin embargo, hay cuestiones que dificultan este proceso, como la incipiente política de valorización del acompañamiento y orientación del preceptor, las insuficiencias del modelo de atención primaria existente para la formación médica, las dificultades en la gestión compartida del proceso y la poca legitimidad de la RM ante la comunidad y los trabajadores del servicio. Conclusión: Hay una relación íntima entre el desarrollo de la red local de salud, en particular de la APS, con la calidad del PRM. Así, es fundamental tener en cuenta no sólo las cuestiones pedagógicas, sino también todo un conjunto de dispositivos de gestión compartida y calificación de la red de servicios.

Palabras clave: Internado y Residencia; Medicina Familiar y Comunitaria; Política de Salud.

\section{Introdução}

A residência médica (RM) é o principal meio de formação médica em nível de pós-graduação, sendo considerada a formação por excelência de especialistas na área médica. ${ }^{1,2}$ É etapa obrigatória na formação do profissional médico em parte significativa dos países, ${ }^{3}$ embora no Brasil essa ainda não seja a realidade. E, embora sejam necessárias evidências científicas mais robustas, é consensual apontar a especialização médica no formato de residência médica como etapa fundamental no sentido de oferecer à sociedade um profissional médico adequado com vistas ao atendimento de suas necessidades. ${ }^{4}$

Isso não é diferente para a prática médica na Atenção Primária à Saúde (APS). Válida para qualquer sistema de saúde, seja universal ou mesmo local, público ou privado, entende-se que a adequação quantitativa e qualitativa da força de trabalho de médicos especialistas em Medicina de Família e Comunidade (MFC) é estratégia salutar para a qualificação da APS. ${ }^{5}$ E sabe-se que uma APS bem estruturada resulta em melhores indicadores de saúde populacionais, maior equidade na atenção à saúde, custos mais favoráveis para a sociedade, coordenação sistêmica mais efetiva e maior segurança para os usuários dos serviços de saúde. ${ }^{6-8}$ 
Assim, tem-se um círculo virtuoso que correlaciona a formação especializada em MFC, o fortalecimento da APS e a qualificação do sistema de saúde, sendo isso algo socialmente necessário e desejável. 3,5,7,9 Não por acaso países desenvolvidos com sistemas de saúde consolidados investem e regulam fortemente seu aparato de especialização médica em nível de residência, destinando parte significativa das vagas para a MFC. ${ }^{3}$

Os primeiros Programas de RM em MFC (PRM-MFC) do Brasil surgiram em 1976, sendo que o respaldo legal para o funcionamento desses programas junto à Comissão Nacional de Residência Médica (CNRM) viria apenas em 1981 e o reconhecimento da especialidade pelo Conselho Federal de Medicina ocorreu muito tempo depois, em 1986.9,10 É importante frisar tais marcos históricos iniciais desse processo de consolidação da formação em MFC e seu reconhecimento enquanto especialidade médica para ilustrar os enormes desafios históricos que acompanham a constituição e expansão da MFC no Brasil desde suas origens.

Contudo, alguns marcos recentes efetivamente promoveram o fortalecimento da especialidade no país. Sem aprofundar na discussão específica sobre cada programa, é possível citar a própria criação da Estratégia de Saúde da Família na década de 1990, que aumentou substancialmente o mercado de trabalho e a relevância da MFC e da APS no país; outros programas do governo federal, como o Programa de Apoio à Formação de Médicos Especialistas em Áreas Prioritárias (Pró-Residência), com oferta de bolsas para RM em todo o país; e o Programa Mais Médicos, que de certa forma buscou interferir na formação médica na direção de uma prática generalista e mais próxima das necessidades do Sistema Único de Saúde (SUS). ${ }^{2}$ Além disso, é importante destacar a opção política feita por diversas gestões municipais pelo fortalecimento de sua rede de APS, o que incluiu maiores investimentos na formação do MFC: maior atratividade profissional, valorização da formação específica em MFC (RM ou Título de Especialista) e a criação de PRM-MFC municipais, a se destacar Rio de Janeiro, Curitiba e Florianópolis. ${ }^{911-13}$

Dessa forma, houve um aumento substancial de vagas para RM em MFC no país nos últimos anos, impactando de maneira positiva o número de especialistas em MFC que entram no mercado a cada ano. ${ }^{13,14}$ Essa expansão dos PRM-MFC aponta para a necessidade de se discutir profundamente os desafios postos à gestão de PRM em nível local ligados à rede municipal de APS. São inúmeras as dificuldades experimentadas nesse processo de formação do MFC, incluindo a construção de estrutura física adequada para o processo de ensino-aprendizagem do residente, a valorização e formação do preceptor médico para a RM, o desenvolvimento das interfaces de trabalho entre gestão e instituições de ensino responsáveis pelos PRM, a melhoria da estrutura de ensino em MFC/APS nas instituições de ensino, o aumento da taxa de ocupação das vagas dos PRM-MFC e de fixação dos residentes na RM e a qualificação de todo o processo de planejamento e execução pedagógica do PRM com vistas à formação ótima do especialista em MFC. ${ }^{9,11-15}$

É nesse sentido que este relato de experiência busca apresentar algumas questões do processo de implantação de três PRM-MFC na cidade de Vitória, capital do Espírito Santo, na perspectiva da interface dos programas com a realidade do município, não sendo objeto específico deste relato as questões pedagógicas da RM. A difusão de tais experiências é salutar na medida em que pode contribuir para o amadurecimento das políticas de formação em MFC no Brasil e pode trazer reflexões importantes para outras localidades que iniciam ou estão consolidando suas próprias experiências formativas. 


\section{Método}

Este relato baseou-se nas experiências dos autores em todo o processo de implantação e consolidação da RM em MFC no município, sendo o grupo composto por gestores municipais do processo de integração ensino-serviço e supervisores dos PRM-MFC situados localmente. Os autores são pessoas profundamente envolvidas com essas questões por longo período de tempo, sendo alguns dos principais responsáveis pela organização do processo de implementação dos PRM-MFC no município. Dessa forma, este relato foi produzido a partir dessas vivências e das narrativas desses distintos sujeitos imersos na realidade descrita. Em momento presencial, as diferentes visões sobre o processo foram confrontadas, amadurecidas e enriquecidas, sendo produzida uma narrativa significativa a todo o grupo. Essa narrativa contempla uma descrição da APS e dos PRM-MFC do município e uma análise sobre os desafios territoriais encontrados durante a implementação dos programas, sob a ótica dos autores do artigo. E para auxiliar essa dinâmica, utilizou-se uma técnica de matriz do tipo SWOT (acrônimo para Strengths, Weaknesses, Opportunities e Threats) para a sistematização dos principais pontos que na visão dos autores influenciam a concretização dos PRM-MFC na rede de serviços municipal.

\section{A Atenção Primária à Saúde no município de Vitória}

Vitória é a capital do Espírito Santo e está localizada na Região Metropolitana de Vitória, conurbação que incorpora outros cinco municípios nos quais reside praticamente metade da população capixaba. Possui população estimada para o ano de 2017, segundo o Instituto Brasileiro de Geografia e Estatística (IBGE), ${ }^{16}$ de 363.140 habitantes e densidade demográfica de 3.761,7 habitantes por quilômetro quadrado. É a cidade com maior salário médio mensal dos trabalhadores formais e quinto maior PIB per capita do estado, apresentando altas taxas de escolaridade e bons indicadores de saúde. Em sentido histórico, Vitória é a cidade onde se instalou a maior parte da rede de alta complexidade em saúde do estado, possuindo uma vasta quantidade de equipamentos especializados e hospitalares públicos e privados. ${ }^{17}$

O município passou por diversas modelagens assistenciais de sua rede de atenção ao longo das décadas, tendo iniciado a implantação da Saúde da Família ainda em 1998 com cinco equipes de Saúde da Família (eSF) e 39 equipes de Agentes Comunitários de Saúde. ${ }^{17}$ Atualmente a organização da atenção à saúde no município de Vitória abrange seis regiões (região 1: Santo Antônio; região 2: Maruípe; região 3: São Pedro; região 4: Forte de São João; região 5: Continental; região 6: Centro) e 29 Territórios de Saúde, o que corresponde ao número de Unidades Básicas de Saúde (UBS). ${ }^{18}$ Das 29 UBS, apenas sete não possuem Estratégia Saúde da Família (ESF), sendo que três destes serviços acomodam equipes de Estratégia de Agentes Comunitários de Saúde (EACS). As 22 Unidades de Saúde da Família (USF) possuíam, em agosto de 2017, 78 eSF em exercício, gerando uma cobertura populacional estimada de aproximadamente $80 \%$, subindo para $88 \%$ quando se considera toda a APS do município. ${ }^{19}$

As USF do município são informatizadas há nove anos e contam com um sistema virtual de gestão plena da atenção à saúde desenvolvido pela equipe de desenvolvimento tecnológico da Secretaria Municipal de Saúde (SEMUS). Esse sistema, denominado Rede Bem-Estar, interliga todos os serviços de saúde do município, incluindo UBS/USF, prontos atendimentos, farmácias, serviços odontológicos e laboratoriais, e centros de referência e de especialidades. Possui um prontuário eletrônico desenvolvido pela mesma equipe 
e integrado a outras ferramentas de gestão: principais Sistemas de Informação em Saúde interligados com a Vigilância em Saúde; agenda de atendimentos dos profissionais; serviços de monitoramento de qualidade da atenção pelo usuário com o uso de mensagens de celular (SMS); e atestados médicos validados pelo QR Code. Em 2017, foi implantado um serviço de agendamento de consultas médicas online (incluindo um módulo disponível para aplicativos para smartphones), integrado a Rede Bem-Estar.

Além disso, as eSF atuam em interface com as equipes de saúde bucal e uma equipe de profissionais de suporte com carga horária semanal de $40 \mathrm{~h}$, incluindo educador físico, psicólogo, farmacêutico, assistente social e, em alguns lugares, fonoaudiólogo e nutricionista. As eSF também contam com o suporte de Apoio Matricial em algumas áreas estratégicas (Saúde do Idoso, Saúde Mental, Saúde da Mulher e Infecções Sexualmente Transmissíveis) e de oito Núcleos de Apoio à Saúde da Família (NASF) tipo I. E foram inseridos links na Rede Bem-Estar para que o profissional acesse diretamente os serviços de teleconsultoria assíncrona do Programa Telessaúde Espírito Santo.

Algo incomum no país, o município conta com uma Escola Técnica do SUS (ETSUS), sendo uma estrutura específica de gestão da interface ensino-serviço-comunidade e do processo de educação permanente profissional. É um espaço de discussão, formulação, articulação e proposição de ações de educação permanente em saúde, a partir do perfil epidemiológico da população, dos processos de organização do cuidado em saúde e da gestão social sobre as políticas públicas de saúde. Dentre os inúmeros projetos e iniciativas gerenciadas pela ETSUS, encontram-se toda a inserção de graduandos da área da saúde em estágios curriculares e atividades práticas nos serviços de saúde municipal (3.019 estudantes frequentaram as UBS em 2017), experiências como o Programa de Educação pelo Trabalho em Saúde (PET-Saúde) ${ }^{20}$ e parte significativa da gestão do planejamento, implementação e monitoramento dos PRM em MFC.

\section{A Residência Médica em Medicina de Família e Comunidade em Vitória}

Em 2017, o município de Vitória contava com três PRM-MFC atuando em seus serviços de APS, em prontos atendimentos e em serviços especializados. As principais características desses três PRM estão descritas na Tabela 1. É importante destacar que o primeiro PRM-MFC do estado funcionou, no período de 1982 a 1996, em um serviço de Medicina Comunitária da Universidade Federal do Espírito Santo localizado no município de Vitória (nessa época, a MFC atendia pelo nome de Medicina Geral e Comunitária). ${ }^{10} \mathrm{O}$ encerramento das atividades desse programa deve-se fundamentalmente à falta de candidatos às vagas ofertadas, o que dentre outros motivos decorreu do ainda incipiente mercado de trabalho para o MFC à época, sendo importante lembrar que a forte expansão do modelo Saúde da Família de APS ocorreria posteriormente. ${ }^{19}$ Novas vagas de residência em MFC só seriam ofertadas no ES quase 20 anos depois.

\section{A gestão dos Programas de Residência Médica em Medicina de Família e Comunidade em Vitória}

A gestão dos PRM-MFC no município ocorre de forma bipartite, considerando que as instituições supervisoras dos programas estabelecem convênios com a secretaria de saúde no sentido de oferecer as vagas de residência à sociedade. Nesse sentido, a atuação das instituições supervisoras nesse processo se materializa em suas respectivas Comissões de Residência Médica (COREME), com as quais se vinculam 
Tabela 1. Características dos Programas de Residência Médica em Medicina de Família e Comunidade que atuam na rede de atenção à saúde do município de Vitória-ES, 2017.

\begin{tabular}{lccc}
\hline & EMESCAM & UFES & Unimed Vitória \\
\hline Ano de criação & 2014 & 2015 & 2015 \\
Natureza da instituição conveniada & Filantrópica & Pública & Privada \\
Número de vagas de R1 & 4 & 3 & 2 \\
Ocupação das vagas de R1 em 2017 (\%) & 75 & 66,6 & 100 \\
Número de egressos até 2017 & 3 & 0 & 0 \\
Número de UBS utilizadas & 2 & 2 & 1 \\
Relação Preceptor (40 h):Residente & $1,5: 4$ & $1: 2$ & $1: 1$ \\
Complementação de bolsa-residente & Não & Não & Não \\
\hline
\end{tabular}

os respectivos coordenadores dos PRM, todos MFC com residência na área. Assim, são as responsáveis pela criação do PRM junto à CNRM e pela sua coordenação, supervisão e avaliação permanente; pela provisão de recursos suficientes para o funcionamento da COREME; pela coordenação do processo seletivo dos residentes; pela emissão do certificado de conclusão do programa; e pela gestão da vida acadêmica dos residentes, incluindo eventuais punições a estes.

Ao município, cabe a gestão plena dos serviços que receberão os residentes; o apoio à coordenação do PRM; a avaliação permanente de seu funcionamento; e a elaboração de políticas que dimensionem todo o arcabouço estrutural e assistencial no qual o residente será inserido. Em Vitória, a ETSUS possui papel fundamental em todo esse processo, contando para isso com articulações com gerências e coordenações do plano assistencial e de vigilância em saúde, bem como de desenvolvimento tecnológico.

Dessa forma, a maior parte da dinâmica de gestão dos PRM ocorre no contínuo diálogo dos supervisores dos programas com a equipe da ETSUS responsável pela residência médica e multiprofissional (Coordenação Geral e Setor de Estágio e Pesquisa). O monitoramento dos PRM se dá no planejamento integrado inicial nos serviços com os gestores, preceptores, supervisores do programa e técnicos da ETSUS; e em reuniões programadas anualmente para avaliação de atividades desenvolvidas, dificuldades identificadas e propostas de ajustes para o ano seguinte. A avaliação das atividades do residente tem especificidades em cada PRM-MFC, com a utilização das diversas metodologias ativas disponíveis, sendo condensada nas reuniões de planejamento integrado, nas sessões anuais e nos múltiplos espaços conjuntos de reuniões e supervisões locais feitas tanto pelas coordenações dos programas quanto pela equipe de gestão municipal.

Como se pode ver, todo esse processo pode encontrar algumas limitações com certo nível de fragmentação da gestão dos PRM-MFC. São três PRM, com suas respectivas e independentes COREME (responsáveis não apenas pelos PRM-MFC, mas inúmeros outros programas voltados para outros níveis de atenção), dialogando com uma SEMUS que possui uma ETSUS como seu principal interlocutor no processo de integração ensino-serviço-comunidade, mas que precisa também inserir nesse processo, com graus distintos de intensidade, outras coordenações e setores que dispõem de algum grau de autonomia na gestão de processos de trabalho que influenciarão nos resultados dos PRM. Essa fragmentação, contudo, não inviabilizou a implementação de qualquer processo junto aos PRM-MFC, talvez devido a 
alguns cuidados que foram tomados ao longo desta trajetória, como criar canais integrados de gestão e também pedagógicos (em 2017, os três PRM-MFC uniram seus momentos teóricos em um único momento na ETSUS).

\section{Avaliação da implantação dos Programas de Residência Médica em Medicina de Família e Comunidade em Vitória}

Apesar das peculiaridades locais, pode-se dizer que os desafios enfrentados na construção dos PRM-MFC situados no município de Vitória se assemelham muito ao observado em todo o país. ${ }^{1,2,9,13,15} \mathrm{O}$ Quadro 1 faz uma síntese das fortalezas e das fragilidades de todo esse processo local a partir de uma matriz do tipo SWOT (acrônimo para Strengths, Weaknesses, Opportunities e Threats) construída a partir das experiências acumuladas dos autores e de reuniões avaliativas feitas com residentes.

Quadro 1. Fatores influentes na implementação dos Programas de Residência Médica em Medicina de Família e Comunidade que atuam na rede de atenção à saúde do município de Vitória-ES, 2017.

\begin{tabular}{|c|c|c|}
\hline & Fortalezas & Fragilidades \\
\hline \multirow{9}{*}{ Interno } & $\begin{array}{l}\text { Estrutura física da maior parte dos serviços, com } \\
\text { organização e higiene adequados. }\end{array}$ & $\begin{array}{l}\text { Heterogeneidade dos serviços em termos de processos } \\
\text { de trabalho e estruturação (p. ex., falta de consultório para } \\
\text { atendimento para todos os residentes). }\end{array}$ \\
\hline & USF acessível à população. & $\begin{array}{l}\text { Contrareferência frágil de outros níveis de atenção, mesmo } \\
\text { com a existência de prontuário eletrônico. }\end{array}$ \\
\hline & $\begin{array}{l}\text { Informatização do serviço e desenvolvimento de sistema } \\
\text { de gestão clínica com prontuário eletrônico (Rede } \\
\text { Bem-Estar). }\end{array}$ & $\begin{array}{l}\text { Sobrecarga de trabalho do preceptor, que possui } \\
\text { agenda de atendimento própria e precisa supervisionar o } \\
\text { residente. }\end{array}$ \\
\hline & $\begin{array}{l}\text { Preceptoria presente em tempo integral e com boa relação } \\
\text { com residente. }\end{array}$ & $\begin{array}{l}\text { Compreensão dos objetivos da residência médica e apoio } \\
\text { ao programa por alguns profissionais do serviço e parte da } \\
\text { comunidade. }\end{array}$ \\
\hline & $\begin{array}{l}\text { Equipe de profissionais ampliada nos serviços, com bom } \\
\text { suporte à equipe de saúde. }\end{array}$ & $\begin{array}{l}\text { Acolhimento do residente no serviço e integração deste } \\
\text { aos processos de trabalho da equipe de saúde. }\end{array}$ \\
\hline & Presença de matriciamento e NASF. & $\begin{array}{l}\text { Suporte insuficiente da gestão para o planejamento das } \\
\text { atividades da residência médica. }\end{array}$ \\
\hline & Existência de uma Escola Técnica do SUS no município. & $\begin{array}{l}\text { Insuficiências estruturais e organizacionais para aumentar } \\
\text { a carteira de serviços das UBS (p. ex., pequenos } \\
\text { procedimentos cirúrgicos). }\end{array}$ \\
\hline & \multicolumn{2}{|l|}{ Gestão municipal apoia o processo. } \\
\hline & Oportunidades & Ameaças \\
\hline \multirow{3}{*}{ Externo } & COREME das instituições que supervisionam os PRM. & Violência nos Territórios de Saúde. \\
\hline & $\begin{array}{l}\text { Experiência exitosa de valorização da preceptoria nos } \\
\text { Hospitais da Rede Estadual de Atenção à Saúde. }\end{array}$ & $\begin{array}{l}\text { Instituições Supervisoras dos PRM com estrutura de ensi- } \\
\text { no em MFC frágeis. }\end{array}$ \\
\hline & Experiências exitosas de PRM-MFC municipais no país. & \\
\hline
\end{tabular}

Em linhas gerais, pode-se dizer que a rede municipal de APS é bem estruturada para sitiar os PRM-MFC. Os serviços possuem boa estrutura física; são informatizados; possuem eSF completas em sua maioria; incorporam profissionais de diversas categorias, ampliando a equipe multiprofissional responsável pelo cuidado da população; e apresenta um forte aparato de gestão participativa, incluindo conselhos de gestão local, que contam com ampla participação dos usuários, e conselhos de diretores de serviços que 
em alguma medida aproximam e legitimam a ESF junto à comunidade. Um destaque do município é o investimento feito no desenvolvimento de uma plataforma online própria que abarca um prontuário eletrônico (a já citada Rede Bem-Estar), o que facilita sobremaneira a integração do residente com o histórico dos usuários dos serviços. É consensual o impacto positivo dessa rede virtual na organização dos processos de trabalho e na qualificação da atenção à saúde, sendo um aspecto que diferencia a rede de serviços do município em relação ao que se faz no conjunto do ES.

Os profissionais que compõem as eSF são em sua grande maioria profissionais estatutários do município (aproximadamente $80 \%$ da força de trabalho da APS é concursada e possui estabilidade no trabalho), embora alguns preceptores incorporados a um PRM-MFC tenham um vínculo temporário com o município complementado por um vínculo formal com a instituição de ensino, sendo todos MFC com RM. A maioria dos profissionais médicos das eSF não possuem formação em nível de RM em MFC ou titulação como especialista na área, embora recentemente as preceptoras que atuam junto ao PRM-MFC da instituição de ensino pública tenha obtido seus títulos de especialistas em MFC. Por outro lado, mesmo sem formação específica, esses profissionais em sua maioria se vinculam com um território por longos períodos de tempos (em muitos casos, por mais de década) e passam por uma série de iniciativas de educação permanente promovidas pelo aparato formativo do município (ETSUS), sendo em geral profissionais com atuação muito sólida em APS.

O município possui uma política específica de preceptoria ${ }^{21}$ e uma relação de longa data com Instituições de Ensino Superior (IES) que inserem estudantes de graduação em campos de estágios nas UBS, o que faz com que haja uma discussão permanente do papel e da valorização da função de preceptoria. Fruto de um movimento de anos, recentemente o município inseriu a atuação em preceptoria em seu Plano de Cargos e Salários, embora ainda não esteja previsto nenhum auxílio financeiro para os profissionais que exercem essa função. Assim, parte significativa dos preceptores que auxiliam os PRM-MFC atua de forma voluntária. Por outro lado, as próprias instituições supervisoras dos PRM-MFC não possuem política específica de valorização dessa atuação, seja por questões burocráticas internas que dificultam a ação com profissionais que não são formalmente vinculados à instituição, seja por prioridades que não levam em consideração a importância desse profissional.

Importante frisar também que o município territorializou sua política docente-assistencial, ${ }^{21}$ inicialmente dando conta das graduações em saúde, mas que reverbera e organiza também a distribuição dos PRM-MFC no território. No início dos anos 2000 , as IES passaram a inserir seus estudantes em serviços localizados em uma região de saúde específica, obedecendo em um primeiro momento uma lógica geográfica (a IES ocupava a região de saúde correspondente a sua localização na cidade). Há exceções a essa normativa, mas em linhas gerais tal dinâmica é potente para a administração de todo o processo de integração ensino-serviço.

Por outro lado, apesar da boa estrutura descrita acima, uma questão a ser equalizada na gestão dos PRM-MFC no município relaciona-se à heterogeneidade operacional dos serviços que recebem residentes (e também graduandos). Em algumas USF, não há consultórios suficientes para que os residentes realizem suas atividades clínicas durante toda a semana. Isso se deve a diversos fatores: subdimensionamento da USF para o número de eSF que nela atua; grande número de profissionais da equipe ampliada que utilizam consultórios regularmente para suas atividades próprias; problemas na gestão da ocupação das salas; e manutenção da agenda do médico preceptor. Tais problemas são amenizados pela composição da 
semana padrão do residente com atividades na comunidade ou que não precisem de um consultório para sua realização, bem como pela inserção do residente em rodízios complementares em ambulatórios de especialidades focais em outros serviços (p. ex., hospitais universitários). Contudo, em algumas situações isso não é suficiente para sanar o problema, pois também afeta o conjunto dos trabalhadores do serviço.

Outra questão a ser enfrentada pelo município e pelas instituições supervisoras é a da preceptoria. Em primeiro lugar e como já discutido, há preceptores que são estatutários do município e recebem residentes de maneira voluntária (opta-se por esse termo porque o profissional tem liberdade de se recusar a realizar esta função, mesmo havendo uma política municipal de preceptoria, convênios entre as instituições e normativas do SUS direcionando todos os serviços públicos como espaços de ensino-aprendizagem) e preceptores temporariamente contratados pelo município e com vínculo formal com a IES. Além disso, há em algumas situações uma sobrecarga de funções sobre o preceptor, já que o mesmo mantém uma rotina de atendimentos a sua população, o que pode prejudicar em certa medida seu suporte ao residente mesmo no caso de compartilhamento de consultas com este e redução do volume de pacientes direcionados especificamente para o preceptor. Tais questões colocam o desafio para a municipalidade refletir sua política de preceptoria no sentido de pensar no conjunto dos PRM-MFC e na qualidade da formação do residente, bem como no bem-estar e valorização do médico da rede de atenção que opta por exercer essa função.

$\mathrm{E}$, não menos importante, observa-se a necessidade de permanentemente trabalhar com a sensibilização e legitimação da dinâmica da RM no conjunto dos trabalhadores do serviço e na comunidade. Uma RM modifica em alguma medida o cotidiano do serviço. Há um novo profissional presente no cotidiano da USF (frequentemente funcionando acima de sua capacidade instalada), com objetivos e propósitos específicos, apesar de não opostos ao da eSF. Além disso, surgirão evidentemente tensionamentos de processos e fluxos cristalizados pelo tempo que podem gerar desconfortos e conflitos entre as partes envolvidas. E pode haver algum estranhamento por parte dos usuários regulares do serviço com determinadas mudanças, mesmo que pequenas. Tais questões exigem contínuo diálogo e capacidade de gestão de conflitos e processos micropolíticos que por vezes não estão maduros o suficiente no processo de implementação do PRM-MFC, sendo necessários investimentos coletivos de cogestão.

A questão do modelo de atenção à saúde das UBS também merece reflexão. Os PRM-MFC, seguindo as orientações da Sociedade Brasileira de Medicina de Família e Comunidade (SBMFC) em seu Currículo Baseado em Competências ${ }^{22}$ para os PRM da área, traz uma perspectiva de prática clínica muito abrangente e resolutiva, atinente ao perfil do MFC em todo o mundo, exigindo das estruturas formativas do especialista uma capacidade de desenvolvimento de competências frequentemente acima do que é possível, dada a estrutura instalada vigente. É comum na ESF se estabelecer processos de trabalho excessivamente programáticos (p. ex., HiperDia, Saúde da Mulher, etc.) e com limitações importantes na carteira de serviços da UBS (p. ex., não realização de procedimentos cirúrgicos, dificuldades para a implantação de DIU, acesso reduzido para pessoas com demandas inespecíficas, etc.). ${ }^{23}$ Esse cenário pode não ser suficiente para a formação adequada do MFC. O problema reside na dificuldade de se problematizar um modelo programático muito forte e enraizado na ESF brasileira com vistas a um modelo que equalize melhor a questão do acesso facilitado ao profissional, a abrangência dos serviços oferecidos à população e a permanente consideração da efetividade e da solidez científica de certas práticas, com as ações de promoção da saúde e de vigilância em saúde. ${ }^{24,25}$ 
É nesse sentido que o Quadro 2 consolida alguns dos desafios a serem enfrentados pelo município e pelas instituições supervisoras para a consolidação da implementação dos PRM-MFC no território.

Quadro 2. Aspectos-chaves a serem enfrentados na implementação dos Programas de Residência Médica em Medicina de Família e Comunidade que atuam na rede de atenção à saúde do município de Vitória-ES, 2017.

\footnotetext{
Adequação dos serviços de saúde: garantia de consultórios para atendimento, dimensionamento adequado do número de eSF para cada serviço, melhoria da estrutura física de determinados serviços, provisão de insumos para aumento da carteira de serviços (abrangência do cuidado), etc.

Formação e valorização da preceptoria, considerando as particularidades de cada instituição supervisora ao mesmo tempo em que se faça uma política de preceptoria considerando o conjunto dos PRM-MFC, incluindo um futuro PRM municipal.

Melhoria dos processos de cogestão da formação em MFC no município, incluindo usuários, diretores de serviço, conjunto dos trabalhadores das USF, preceptores, gestores e professores das IES.

Gestão clínica e dos processos de trabalho das eSF, com adequação das agendas de atendimento, reflexão sobre o modelo de atenção à saúde vigente e fortalecimento dos atributos essenciais da APS, principalmente a integralidade do cuidado.

Maior integração entre os PRM-MFC e destes com a graduação médica (estágios realizados nos mesmos locais onde ocorre a RM).

Abordagem de problemas sociais que interferem no trabalho não apenas dos residentes, mas de toda a USF, particularmente da violência social.
}

\section{Considerações Finais}

A grande necessidade de médicos especialistas em MFC no Brasil, tanto para o sistema público quanto para a saúde suplementar, e a recente expansão de vagas de RM na especialidade trazem enormes desafios para IES, gestões municipais, estaduais e federal, bem como para todas as instituições que possuem PRM (p. ex., cooperativas médicas, como no caso analisado neste relato de experiência), para a implementação e qualificação do aparato formador em MFC.

Este relato de experiência apresentou algumas questões relacionadas à implementação de PRM-MFC em serviços de APS de uma capital de estado com altos índices de desenvolvimento social e significativa rede de atenção à saúde dos mais diversos níveis de complexidade tecnológica. Essa experiência aponta na direção de uma íntima relação entre o desenvolvimento da rede local de saúde, em particular da APS, com a qualidade do PRM. Além disso, é importante frisar como esses aspectos também têm o potencial de influenciar a graduação médica com vistas a uma formação mais próxima dos princípios da APS e possivelmente uma maior taxa de escolha pela MFC pelos estudantes. ${ }^{26}$

Aspectos como estrutura do serviço; modelo de atenção e princípios basilares do cuidado à população; concepção de APS e MFC dos envolvidos na implementação do PRM; vínculo do profissional de saúde que atua como preceptor com a municipalidade e com o PRM-MFC; organização do processo de trabalho da eSF; integração das instituições supervisoras do PRM com o município; e dispositivos de gestão compartilhada ensino-serviço-comunidade, influenciam diretamente no resultado dos esforços de criação e execução de um PRM em MFC.

É fundamental que as IES ou qualquer instituição que planeja implementar um PRM-MFC em parceria com uma gestão municipal de saúde tenha em vista não apenas as questões pedagógicas e burocráticas diretamente relacionadas às exigências da CNRM. Grandes esforços conjuntos são necessários no sentido de desenvolver um ambiente propício para a formação qualificada de MFC em acordo com as diretrizes nacionais e a necessidade da população brasileira. A formação de um MFC é complexa e diversificada, 
contemplando uma enorme quantidade de competências com vistas a garantir uma atenção à saúde altamente resolutiva, humanizada e abrangente. Para isso, são necessários investimentos permanentes na qualificação dos serviços de saúde em que o PRM será inserido, no desenvolvimento do corpo técnico responsável pelo acompanhamento cotidiano do residente e na construção de dispositivos de cogestão adequados. Vitória possui uma estrutura forte nesse sentido e mesmo assim não são poucos os problemas encontrados. Dessa forma, é preocupante o estado atual de precarização dos sistemas locais de saúde.

\section{Referências}

1. Berger CB, Dallegrave D, Castro Filho ED, Pekelman R. A formação na modalidade Residência Médica: contribuições para a qualificação e provimento médico no Brasil. Rev Bras Med Fam Comunidade. 2017;12(39):1-10. DOI: http://dx.doi.org/10.5712/rbmfc12(39)1399

2. Alessio MM, Sousa MF. Regulação da formação de especialistas: inter-relações com o Programa Mais Médicos. Physis. 2016;26(2):633-67. DOI: http://dx.doi.org/10.1590/S0103-73312016000200015

3. Vicente VC, Pitz PB, Arenal JMC, Ferrández ES, Gonzales FS. La Medicina familiar y comunitaria y la universidad. Informe SESPAS 2012. Gac Sanit. 2012;26(Supl.):69-75. DOI: http://dx.doi.org/10.1016/j.gaceta.2011.07.018

4. van der Leeuw RM, Lombarts KMJMH, Arah OA, Heineman MJ. A systematic review of the effects of residency training on patient outcomes. BMC Medicine. 2012;10:65. DOI: http://dx.doi.org/10.1186/1741-7015-10-65

5. Macinko J, Starfield B, Shi L. Quantifying the health benefits of primary care physician supply in the United States. Int J Health Serv. 2007;37(1):111-26. DOI: http://dx.doi.org/10.2190/3431-G6T7-37M8-P224

6. World Health Organization. The world health report 2008 - Primary health care: Now more than ever. World Health Organization; 2008.

7. Starfield B. Atenção Primária: equilíbrio entre necessidades de saúde, serviços e tecnologia. Brasília: Unesco, Ministério da Saúde;2002.

8. Macinko J, Starfield B, Shi L. The contribution of primary care systems to health outcomes within Organization for Economic Cooperation and Development (OECD) countries, 1970-1998. Health Serv Res. 2003;38(3):831-65. DOI: http://dx.doi.org/10.1111/1475-6773.00149

9. Justino ALA, Oliver LL, Melo TP. Implantação do Programa de Residência em Medicina de Família e Comunidade da Secretaria Municipal de Saúde do Rio de Janeiro, Brasil. Ciênc. saúde coletiva. 2016;21(5):1471-80. DOI: http://dx.doi.org/10.1590/1413-81232015215.04342016

10. Falk JW. A Medicina de Família e Comunidade e sua entidade nacional: histórico e perspectivas. Rev Bras Med Fam Comunidade. 2004;1(1):5-10. DOI: http://dx.doi.org/10.5712/rbmfc1(1)2

11. Poli Neto P.O Programa de Residência em Medicina de Família e Comunidade do município de Curitiba. Rev Bras Med Fam Comunidade. 2014;9(31):192-4. DOI: http://dx.doi.org/10.5712/rbmfc9(31)904

12. Lermen Jr N. O Programa de Residência em Medicina de Família e Comunidade do município de Florianópolis. Rev Bras Med Fam Comunidade. 2014;9(32):300-3. DOI: http://dx.doi.org/10.5712/rbmfc9(32)992

13. Storti MMT, Oliveira FP, Xavier AL. A expansão de vagas de residência de Medicina de Família e Comunidade por municípios e o Programa Mais Médicos. Interface (Botucatu). 2017;21(Suppl 1):1301-14. DOI: http://dx.doi.org/10.1590/1807-57622016.0511

14. Trindade TG. Residência Médica em Medicina de Família e Comunidade [palestra]. Ministério da Educação. 25 Agosto 2015.

15. Castells MA, Campos CEA, Romano VF. Residência em Medicina de Família e Comunidade: atividades da preceptoria. Rev. bras. educ. med. 2016;40(3):461-69. DOI: http://dx.doi.org/10.1590/1981-52712015v40n3e02862014

16. Instituto Brasileiro de Geografia e Estatística. Panorama. [acesso em 2018 jan 30]. Disponível em: https://cidades.ibge.gov.br/brasil/es/ vitoria/panorama

17. Siqueira MP. Regulação assistencial e integralidade na assistência à saúde em Vitória, sob a perspectiva da Análise Institucional [dissertação]. Vitória: Universidade Federal do Espírito Santo; 2010.

18. Prefeitura de Vitória. Plano Municipal de Saúde: 2014-2017. Vitória; 2013.

19. Ministério da Saúde. Histórico de cobertura da Saúde da Família. Brasília: Departamento de Atenção Básica; 2018c. [acesso em 2018 jan 30]. Disponível em: http://dab.saude.gov.br/portaldab/historico_cobertura_sf.php 
20. Dias HS, Lima LD, Teixeira M. A trajetória da política nacional de reorientação da formação profissional em saúde no SUS. Ciênc. saúde coletiva. 2013;18(6):1613-24. DOI: http://dx.doi.org/10.1590/S1413-81232013000600013

21. Secretaria Municipal de Saúde de Vitória. Manual de orientação das práticas de integração ensino serviço no município de Vitória - ES. Vitória: ETSUS; 2013. [acesso em 2018 jan 5]. Disponível em: http://m.vitoria.es.gov.br/arquivos/20100222_manual_praticas.pdf

22. Sociedade Brasileira de Medicina de Família e Comunidade. Currículo baseado em competências para Medicina de Família e Comunidade. Rio de Janeiro; 2014.

23. Sarti TD. A (Bio) política da Saúde da Família: adoecimento crônico, micropolítica do trabalho e o governo da vida [tese]. São Paulo: Faculdade de Saúde Pública, Universidade de São Paulo; 2015. DOI: http://dx.doi.org/10.11606/T.6.2015.tde-16072015-113309

24. Tesser CD, Norman AH. Repensando o acesso ao cuidado na Estratégia Saúde da Família. Saude soc. 2014;23(3):869-83. DOI: http://dx.doi.org/10.1590/S0104-12902014000300011

25. Norman AH, Tesser CD. Acesso ao cuidado na Estratégia Saúde da Família: equilíbrio entre demanda espontânea e prevenção/promoção da saúde. Saude soc. 2015;24(1):165-79. DOI: http://dx.doi.org/10.1590/S0104-12902015000100013

26. Dornan T, Littlewood S, Margolis SA, Scherpbier A, Spencer J, Ypinazar V. How can experience in clinical and community settings contribute to early medical education? A BEME systematic review. Med Teach.2006;28(1):3-18. DOI: http://dx.doi.org/10.1080/01421590500410971 